\title{
Bénard-von Kármán Vortex Street in a Bose-Einstein Condensate
}

\author{
Kazuki Sasaki, Naoya Suzuki, and Hiroki Saito \\ Department of Engineering Science, University of Electro-Communications, Tokyo 182-8585, Japan
} (Received 10 February 2010; revised manuscript received 18 March 2010; published 16 April 2010)

\begin{abstract}
Vortex shedding from an obstacle potential moving in a Bose-Einstein condensate is investigated. Longlived alternately aligned vortex pairs are found to form in the wake, which is similar to the Bénardvon Kármán vortex street in classical viscous fluids. Various patterns of vortex shedding are systematically studied and the drag force on the obstacle is calculated. It is shown that the phenomenon can be observed in a trapped system.
\end{abstract}

DOI: 10.1103/PhysRevLett.104.150404

The formation of a train of alternate vortices in the wake past an obstacle, known as the Bénard-von Kármán vortex street, is a ubiquitous and intriguing phenomenon in fluids. Since the pioneering experimental study by Bénard [1] and theoretical consideration by von Kármán [2], numerous studies have been made on the phenomena of vortex street formation [3].

The behavior of a viscous fluid flowing past an obstacle is determined by the Reynolds number Re, which is a dimensionless parameter that includes the kinematic viscosity. The wake of a cylinder is steady for Re below around 50, and a vortex street emerges for $10^{2} \leqslant \operatorname{Re} \lesssim$ $10^{5}$, which becomes turbulence for a larger Re. This implies that the viscosity plays an important role in vortex street formation in classical fluids. For superfluids, however, the Reynolds number cannot be defined because of the absence of viscosity. Moreover, the vortex quantization makes superfluid dynamics quite different from classical fluid dynamics. Therefore, it is not obvious whether the instability of the wake and subsequent vortex street generation occur in superfluids. According to von Kármán's theory $[2,4]$, a vortex street is expected to be very longlived in inviscid fluids, once it is created.

In this Letter, by numerically solving the GrossPitaevskii (GP) equation, we show that long-lived alternately aligned vortex pairs are formed in the wake of an obstacle potential moving in a Bose-Einstein condensate (BEC), which is similar to the vortex street in classical fluids. Mean-field analysis of systems of a BEC with a moving potential has been performed by many authors from the viewpoints of drag force [5-7], vortex dynamics near the cylinder [8,9], critical velocity [10], scaling laws [11], supersonic flows [12,13], and multicomponent systems $[14,15]$. However, vortex street formation was not found in these studies, probably because the parameter region for which a vortex street emerges is narrow, as shown later.

We consider a BEC of atoms with mass $m$ and an obstacle potential $V$ moving in the $-x$ direction at a velocity $v$. In the mean-field theory, the condensate is described by the macroscopic wave function $\psi$ obeying the GP equation given by
PACS numbers: 05.30.Jp, 03.75.Kk, 03.75.Lm, 67.85.-d

$$
i \hbar \frac{\partial \psi}{\partial t}=-\frac{\hbar^{2}}{2 m} \nabla^{2} \psi+V \psi+g|\psi|^{2} \psi,
$$

where $g=4 \pi \hbar^{2} a / m$ with $a$ being the $s$-wave scattering length of the atoms. We employ a Gaussian potential with peak strength $V_{0}$ and radius $d$ moving in the $-x$ direction at a velocity $v$ as $V=V_{0} \exp \left\{-\left[(x+v t)^{2}+y^{2}\right] / d^{2}\right\}$. Normalizing space and time by $\hbar /\left(m g n_{0}\right)^{1 / 2}$ and $\hbar /\left(g n_{0}\right)$, where $n_{0}$ is the atom density without perturbation, we can eliminate the interaction parameter $g$ from Eq. (1). We numerically solve Eq. (1) in two dimensions under the periodic boundary condition using the pseudospectral method. The initial state is the stationary state of Eq. (1) with $v=0$ plus a small amount of noise to break the symmetry.

Figure 1 shows typical wakes flowing past an obstacle potential with $V_{0} /\left(g n_{0}\right)=100$. For a sufficiently small velocity $v$, the flow around the obstacle is a steady laminar flow and no quantized vortex is created. When the velocity $v$ exceeds a critical velocity, which depends on the interaction strength and the shape of the potential, vortexantivortex pairs are created [5]. The critical velocity for the vortex creation is of the order of the speed of sound $\left(g n_{0} / m\right)^{1 / 2}$. When created vortices separate from the potential, the flow velocity around the potential again exceeds the critical velocity and subsequent vortices are created. A train of vortex-antivortex pairs is thus generated behind the potential. Since a symmetric double row of vortices is unstable [4], the vortex pairs are dislocated sinuously as shown in Fig. 1(a). Nore et al. [16] showed that such a staggered vortex pattern is formed if a double row of vortices is prepared with an appropriate perturbation. Since a pair of point vortices with circulations $\pm h / m(h$ : Planck's constant) and distance $d$ moves in the direction perpendicular to a line between the pair at a velocity $\hbar /(m d)$ [4], the alternately inclined vortex pairs move in two directions [white arrows in Fig. 1(a)], forming a $V$-shaped wake as in Fig. 1(a). The divergence of the wake is significant for large $v$ with small $d$, which forms a pattern similar to supersonic flow $[12,13]$.

Figure 1(b) shows the main result of this study. The significant difference from Fig. 1(a) is that the vortices in 


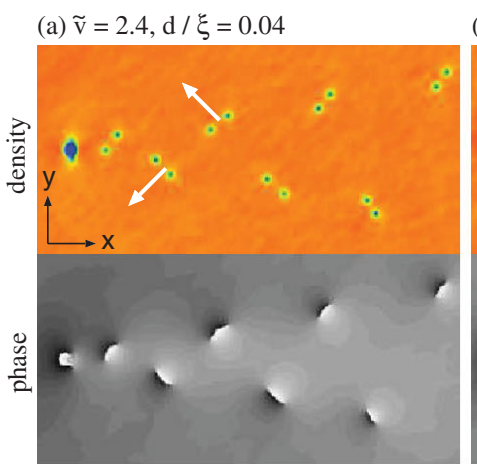

(b) $\tilde{\mathrm{v}}=2.6, \mathrm{~d} / \xi=0.05$

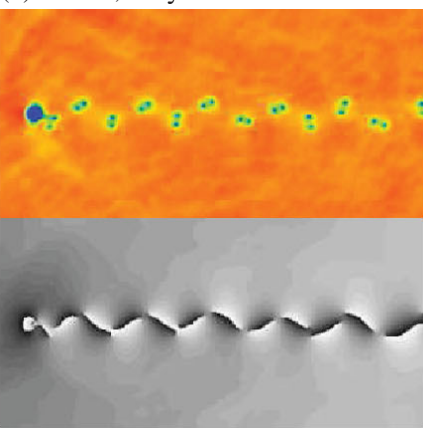

(c) $\tilde{\mathrm{v}}=3.0, \mathrm{~d} / \xi=0.05$

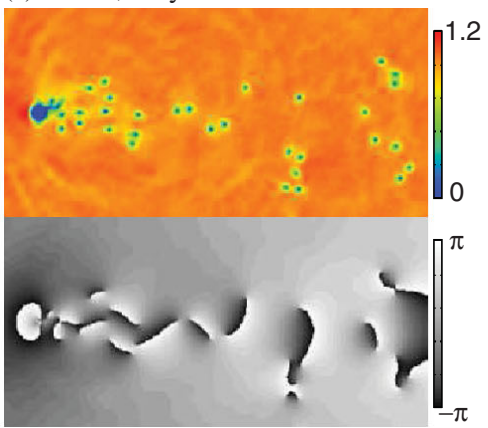

FIG. 1 (color). Density and phase distributions of a condensate past an obstacle potential. The velocity and potential width are $(\tilde{v}, d / \xi)=(2.4,0.04)$ in (a), (2.6,0.05) in (b), and (3.0,0.05) in (c), where $\tilde{v}=v\left[10^{3} m /\left(g n_{0}\right)\right]^{1 / 2} /(2 \pi)$ and $\xi=\hbar\left[10^{3} /\left(m g n_{0}\right)\right]^{1 / 2}$. The white arrows in (a) indicate the directions in which the vortex-antivortex pairs move. The density is normalized by $n_{0}$. The field of view is $6 \xi \times 3 \xi$. In the numerical calculation, a $32 \xi \times 8 \xi$ space is discretized into $4096 \times 1024$.

a pair created by the obstacle potential at a time have the same circulation. Since two point vortices having the same circulation $h / m$ rotate around their center at an angular frequency $2 \hbar /\left(m d^{2}\right)$ without changing their distance [4], the created vortex pairs in Fig. 1(b) remain bound and rotate. The pairs with opposite circulations are alternately released from the obstacle potential to form a train of vortex pairs, resembling a Bénard-von Kármán vortex street [17]. In contrast to the vortex arrangement originally considered by von Kármán, in which isolated point vortices are aligned, the vortex pairs constitute the vortex street in the present case. The detailed dynamics of the vortex street formation just behind the obstacle potential is shown in Fig. 2. The pairs of vortices are released obliquely

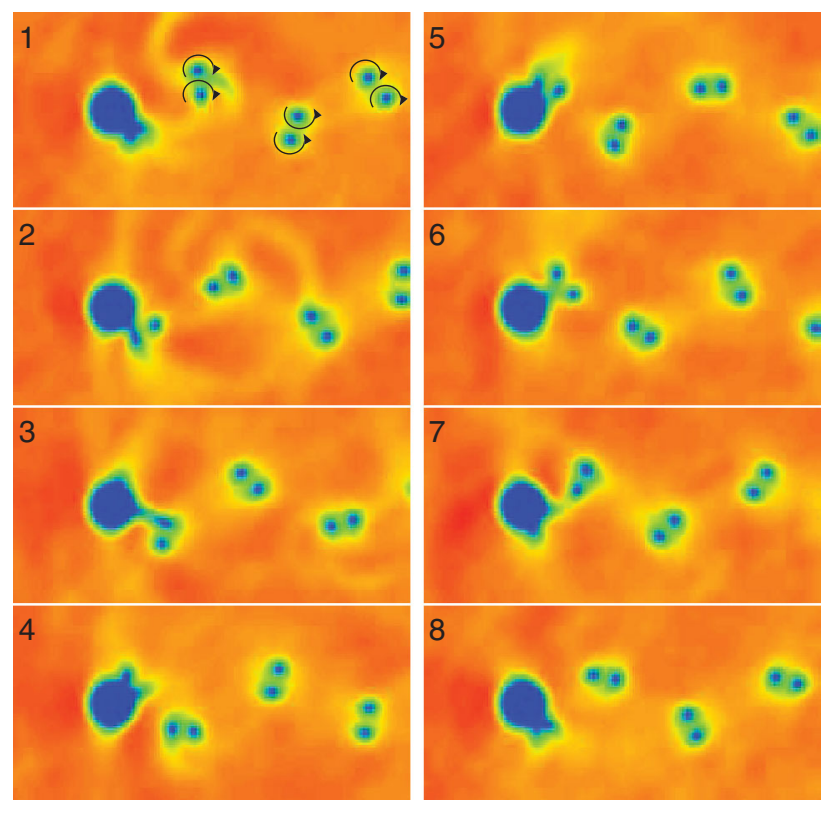

FIG. 2 (color). Serial snapshots of the density profiles for the parameters in Fig. 1(b) in the frame moving with the potential. The time interval is $10 \hbar /\left(g n_{0}\right)$. The arrows indicate the directions of circulations. The field of view is $2 \xi \times \xi$. The color scale is the same as that in Fig. 1. backward left and right with alternate circulations. We find from Figs. 1(b) and 2 that the distance between the two vortex rows is $b \simeq 0.24 \xi$ and the distance between two pairs in a row is $\ell \simeq 0.87 \xi$ on average, and hence $b / \ell \simeq 0.28$, where $\xi=\hbar\left[10^{3} /\left(m g n_{0}\right)\right]^{1 / 2}$. This ratio is in good agreement with the stability condition of von Kármán's vortex arrangement $b / \ell=\pi^{-1} \cosh ^{-1} \sqrt{2} \simeq$ 0.28 [4]. In fact, the vortex street in Fig. 1(b) survives at least $t>10^{3} \hbar /\left(g n_{0}\right)$. The vortex street in Fig. 1(b) moves in the $-x$ direction at a velocity $\simeq 0.14\left(g n_{0} / m\right)^{1 / 2} \simeq$ $0.8 h /(\sqrt{2} \ell m)$. The velocity of von Kármán's point vortices, in which each vortex has a circulation $2 \mathrm{~h} / \mathrm{m}$, is $h /(\sqrt{2} \ell m)$ [4]. For large $d$ and $v$, the periodicity in the wake seems to disappear [Fig. 1(c)].

We systematically performed numerical simulations for various values of $d$ and $v$ to determine the parameter regions for the types of wakes in Fig. 1. Figure 3 shows a rough sketch of each parameter region. The regions of the

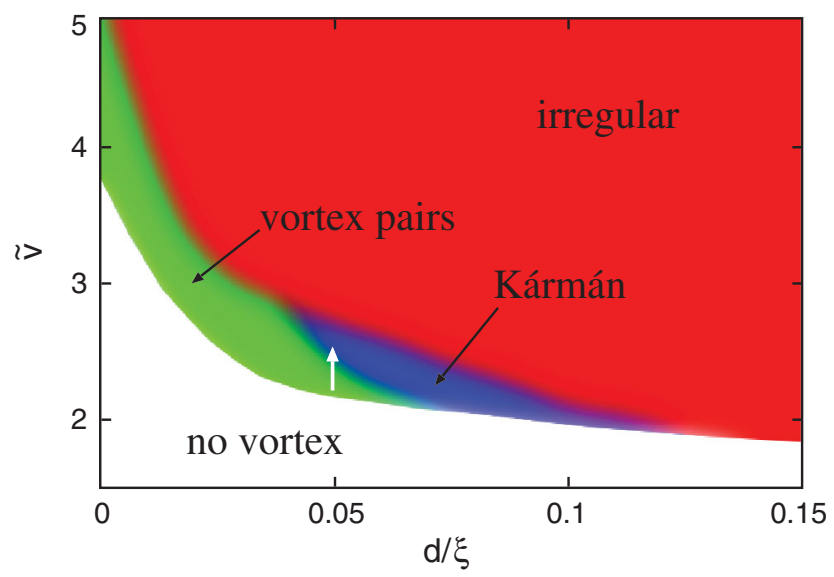

FIG. 3 (color). Dependence of the patterns of wakes on the normalized Gaussian width $d / \xi$ and velocity $\tilde{v}$ of the potential. The green, blue, and red regions correspond to the flow patterns shown in Figs. 1(a)-1(c), respectively. The white region corresponds to stationary laminar flow. The white arrow indicates the change of $\tilde{v}$ used in Fig. 4. 
periodic behaviors shown in Figs. 1(a) and 1(b) are located between the regions of steady laminar flow (white region in Fig. 3) and irregular flow (red). We note that the parameter region for vortex street formation is rather restricted, $0.04 \lesssim d / \xi \lesssim 0.13$ and $1.9 \lessgtr \tilde{v} \lesssim 2.8$, where $\tilde{v}=$ $v\left[10^{3} \mathrm{~m} /\left(g n_{0}\right)\right]^{1 / 2} /(2 \pi)$. This is in contrast with classical fluids, in which the vortex street emerges for a wide range of Reynolds number.

Figure 4 shows gradual change from the vortexantivortex pair creation to the vortex street formation, where $d / \xi=0.05$ is fixed and $v$ is gradually increased as shown in the white arrow in Fig. 3. In Figs. 4(a) and 4(b), the velocity corresponds to the green region in Fig. 3 and vortex-antivortex pairs are created. In Fig. 4(c), $\tilde{v}$ crosses to the blue region in Fig. 3 and the vortex creation $\cdots(+-)(-+)(+-)(-+) \cdots$ gradually changes to $\cdots+)(--)(++)(--)(+\cdots$, resulting in the vortex street formation in Fig. 4(d), where + and - indicate the clockwise and counterclockwise circulations of vortices. Such a sequence of vortex shedding was also found in Ref. [11]. The change from Figs. 4(a) to 4(d) with an increase in $\tilde{v}$ is quite different from the behavior in classical viscous fluids, in which a pair of eddies in the wake becomes unstable and grows into a vortex street in the downstream region.

Figure 5 shows the drag force on the obstacle potential given by $\boldsymbol{F}=\partial_{t} \int d \boldsymbol{r} \psi^{*}(i \hbar \nabla) \psi$. The initial state is the stationary state for $v=0$. At $t=0$ the potential starts to move at a velocity $v$. Figure 5(a) corresponds to the vortexantivortex pair creation in Fig. 1(a). For $\tilde{t} \lesssim 200, F_{x}$ oscillates while $F_{y} \simeq 0$, indicating that the vortex-antivortex pairs are shed from the potential symmetrically. For $\tilde{t} \gtrsim$ 200, the vortex pairs begin to incline as in Fig. 1(a), and $F_{y}$ also starts to oscillate. Figure 5(b) corresponds to the vortex street formation, where both $F_{x}$ and $F_{y}$ oscillate

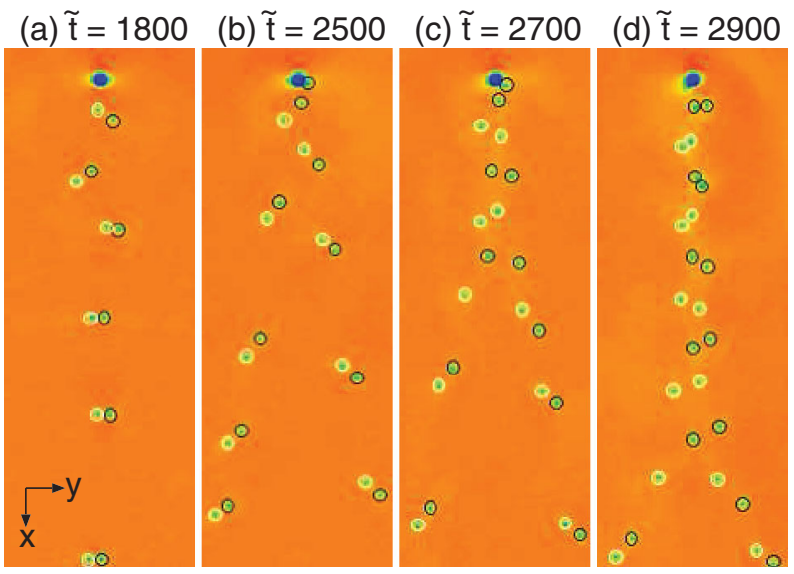

FIG. 4 (color). Snapshots of the density profiles for $d / \xi=$ 0.05. The velocity is $\tilde{v}=2.2$ for $\tilde{t}<1000$ and $\tilde{v}=$ $2.2+0.4(\tilde{t}-1000) / 4000$ for $\tilde{t}>1000$. The black and white circles indicate clockwise and counterclockwise circulations, respectively. The field of view is $3 \xi \times 8 \xi$. The color scale is the same as that in Fig. 1. for $\tilde{t} \gtrsim 200$. The oscillation in $F_{y}$ in Figs. 5(a) and 5(b) is due to the alternate shedding of vortices and hence its frequency is half the vortex shedding frequency, i.e., the frequency of $F_{x}$. It is interesting to note that $F_{y}$ in Fig. 5(c) oscillates, even though Fig. 1(c) does not seem to have periodicity. A similar phenomenon is also observed in classical fluids with a large Reynolds number [18].

Next, we perform full three-dimensional simulation for a realistic system confined in a trapping potential. A BEC stirred by a moving obstacle potential has been studied experimentally in Refs. [19-21]. We consider a situation in which a BEC of ${ }^{87} \mathrm{Rb}$ with the number of atoms $N=2.1 \times$ $10^{6}$ is confined in a harmonic potential $m\left(\omega_{x}^{2} x^{2}+\omega_{y}^{2} y^{2}+\right.$ $\left.\omega_{z}^{2} z^{2}\right) / 2$ with $\left(\omega_{x}, \omega_{y}, \omega_{z}\right)=2 \pi \times(56,350,4.3) \mathrm{Hz}$. This is the same condition as in the experiment performed by the Berkeley group [22]. The obstacle potential is produced by a blue-detuned Gaussian laser beam along the $y$ direction with $d=1.5 \mu \mathrm{m}$ and $V_{0}=100 \hbar \omega_{x}$, which is initially located at $z=-100 \mu \mathrm{m}$ and $x=0$ at $t=0$ and moves in the $z$ direction at a velocity $v=0.76 \mathrm{~mm} / \mathrm{s}$ for $t>0$. The $s$-wave scattering length is assumed to be $100 a_{B}$ with $a_{B}$ being the Bohr radius. Figure 6 shows the column density integrated along $y$, the phase profiles on the $y=0$ plane, and the isodensity surface at $t=160 \mathrm{~ms}$. We find that a vortex street is generated behind the moving potential, as in Fig. 1(b), which confirms that our finding can be experimentally observed in an inhomogeneous three-dimensional system. The ratio $b / \ell$ in Fig. 6 is about 0.28 , which agrees with the stable condition of the vortex street.

We have numerically confirmed that an impenetrable disk-shaped potential $(V=\infty$ for $r<d$ and $V=0$ for

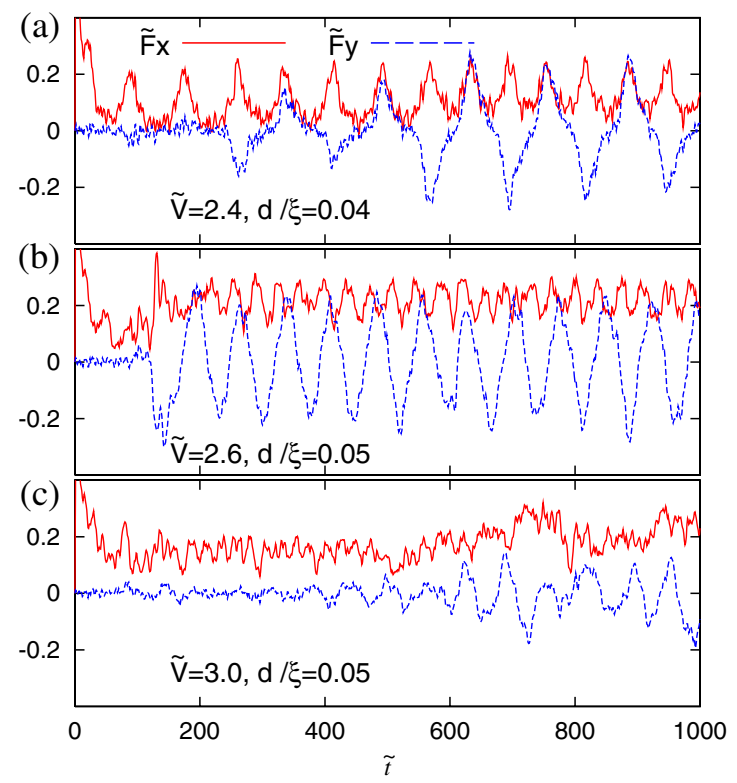

FIG. 5 (color online). Time evolution of the normalized drag force $\tilde{\boldsymbol{F}}=2 \boldsymbol{F} /\left(A m n_{0} \boldsymbol{v}^{2}\right)$, where $A$ is the projected area of the obstacle potential. The solid and dashed lines show $\tilde{F}_{x}$ and $\tilde{F}_{y}$. The parameters used in (a)-(c) are the same as those in Figs. 1(a)-1(c), respectively. 


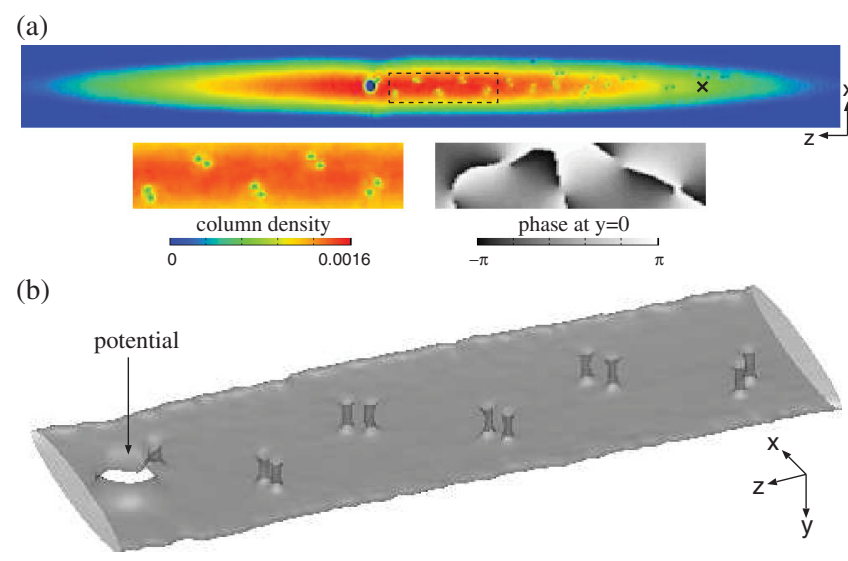

FIG. 6 (color). A BEC of ${ }^{87} \mathrm{Rb}$ atoms confined in a harmonic potential at $t=160 \mathrm{~ms}$, where the Gaussian potential is initially located at the marked position $(\times)$ and starts to move in the $z$ direction at a constant velocity. (a) Column density normalized by $N m \omega_{x} / \hbar$. The dashed region is magnified in the lower panels. The gray scale image shows the phase at $y=0$. The field of view is $300 \times 30 \mu \mathrm{m}$ for the main panel and $40 \times 10 \mu \mathrm{m}$ for the lower panels. (b) Translucent display of the isodensity surface for the state shown in (a).

$r>d$ ) also generates vortex patterns similar to those in Fig. 1. This potential can be realized by a flattop laser beam or a Gaussian beam in which limit $V_{0} \rightarrow \infty$ and $d \rightarrow 0$ is taken with $d^{2} \log \left(V_{0} / \mu\right)$ being fixed, where $\mu$ is the chemical potential.

A future prospect of this study is to provide a mathematical description of the alternate creation of the vortex pairs as shown in Fig. 2. From an experimental point of view, it is important to study the cases of various trapping potentials including cigar-shaped and oblate traps. Various shapes of obstacle potentials also merit further study.

In conclusion, we have shown that pairs of quantized vortices shed from an obstacle potential moving in a BEC alternately align and survive for a long time [Figs. 1(b) and 2], which closely resembles the Bénard-von Kármán vortex street in classical fluids. We have obtained the parameter region in which a vortex street emerges (Fig. 3) and calculated the drag force (Fig. 5). We have shown that vortex street formation can be observed in a trapped BEC disturbed by a blue-detuned laser beam (Fig. 6). Since the vortex street generation typifies the great diversity of classical fluid dynamics, the emergence of the vortex street in a BEC implies that a rich variety of phenomena is still unrevealed in quantum hydrodynamics.

We thank Takeshi Miyazaki and Naoya Takahashi for their valuable comments. This work was supported by
KAKENHI from MEXT (No. 17071005 and No. 20540388).

[1] H. Bénard, C. R. Acad. Sci. Paris Ser. IV 147, 839 (1908); 147, 970 (1908).

[2] T. von Kármán, Nachr. Ges. Wiss. Göttingen, Math. Phys. K1. 509 (1911); 547 (1912).

[3] For review, see, for example, C. H. K. Williamson, Annu. Rev. Fluid Mech. 28, 477 (1996).

[4] For example, H. Lamb, Hydrodynamics (Dover, New York, 1945), 6th ed., Chap. VII.

[5] T. Frisch, Y. Pomeau, and S. Rica, Phys. Rev. Lett. 69, 1644 (1992).

[6] T. Winiecki, J. F. McCann, and C. S. Adams, Phys. Rev. Lett. 82, 5186 (1999).

[7] T. Winiecki, B. Jackson, J. F. McCann, and C. S. Adams, J. Phys. B 33, 4069 (2000).

[8] C. Nore, C. Huepe, and M. E. Brachet, Phys. Rev. Lett. 84, 2191 (2000).

[9] A. Aftalion, Q. Du, and Y. Pomeau, Phys. Rev. Lett. 91, 090407 (2003).

[10] J. S. Stießberger and W. Zwerger, Phys. Rev. A 62, 061601 (R) (2000).

[11] C. Huepe and M. E. Brachet, Physica (Amsterdam) 140D, 126 (2000).

[12] G. A. El, A. Gammal, and A. M. Kamchatnov, Phys. Rev. Lett. 97, 180405 (2006).

[13] I. Carusotto, S. X. Hu, L. A. Collins, and A. Smerzi, Phys. Rev. Lett. 97, 260403 (2006).

[14] H. Susanto, P. G. Kevrekidis, R. Carretero-González, B. A. Malomed, D. J. Frantzeskakis, and A. R. Bishop, Phys. Rev. A 75, 055601 (2007).

[15] A. S. Rodrigues, P. G. Kevrekidis, R. Carretero-González, D. J. Frantzeskakis, P. Schmelcher, T. J. Alexander, and Yu. S. Kivshar, Phys. Rev. A 79, 043603 (2009).

[16] C. Nore, M.E. Brachet, and S. Fauve, Physica (Amsterdam) 65D, 154 (1993).

[17] We note that the classical vortex street formation is typically discussed for incompressible fluids, while the release of vortices in a BEC is connected with compressibility.

[18] A. Roshko, J. Fluid Mech. 10, 345 (1961).

[19] C. Raman, M. Köhl, R. Onofrio, D. S. Durfee, C.E. Kuklewicz, Z. Hadzibabic, and W. Ketterle, Phys. Rev. Lett. 83, 2502 (1999).

[20] R. Onofrio, C. Raman, J. M. Vogels, J. R. Abo-Shaeer, A. P. Chikkatur, and W. Ketterle, Phys. Rev. Lett. 85, 2228 (2000).

[21] T. W. Neely, E. C. Samson, A. S. Bradley, M. J. Davis, and B. P. Anderson, arXiv:0912.3773.

[22] L. E. Sadler, J. M. Higbie, S. R. Leslie, M. Vengalattore, and D. M. Stamper-Kurn, Nature (London) 443, 312 (2006). 